\title{
Les soies infra-internes des palpes chez les Ixodidae
}

\author{
par G. SENEVET \\ Laboratoire de Parasitologie et de Mycologie ( $\mathrm{P}^{\mathrm{r}} \mathrm{M}$. LARIVIÈre) \\ U.E.R. des Cordeliers, 15, rue de l'Ecole-de-Médecine, F 75 -Paris $\left(6^{\circ}\right)$
}

\begin{abstract}
Résumé
$S$. définit, les soies infra-internes des palpes dont il indique la morphologie et la localisation.

A l'aide de divers tableaux comparatifs, il montre que ces soies sont plus nombreuses chez les adultes que chez les nymphes et surtout que chez les larves.

Le sexe ne semble pas avoir d'importance.

Ces soies font complètement défaut à l'article I dans certains genres (Ixodes).

La variabilité de leur forme et de leur nombre, permettra peut-être de les utiliser dans la diagnose.
\end{abstract}

\section{Summary}

$S$, defines the infra-internal bristles of the palps.

He describes the morphology and localization.

By means of some comparative tables, he shows the greater number in adults than in pupae and specially larvae.

No great difference between the sexes.

The infr. int. bristles are entirely lacking on segment $\mathrm{I}$, in genus Ixodes.

The variability of their shape and of their number will perhaps enable us to use their in the diagnosis.

Sous le nom de soies infra-internes («soies ventrales internes » de quelques auteurs) on désigne un groupe de soies des palpes, visibles par la face ventrale et situées sur le côté interne du palpe entre celui-ci et le bord externe de l'hypostome. 
Chez les adultes, on trouvera, en principe, une ou plusieurs soies sur chacun des articles I, II et II. (Chez les Argasidés, que nous comptons étudier ultérieurement, on les trouvera de même sur les articles I, II, III et IV).

L'article II est le plus fréquent des porteurs.

Chez les nymphes, la répartition varie avec les genres, mais les soies sont moins nombreuses que chez les adultes. De même chøz les larves où le nombre est très réduit : par exemple à un ou plus rarement deux sur l'article II, et où l'article I en est toujours dépourvu.

L'intérêt de ces soies paraît avoir échappé à pas mal d'auteurs. C'est ainsi qu'elles ne sont ni signalées ni figurées dans certaines publications d'Arthur, de de Babos, de Delpy, ainsi que dans les «Tiques du Soudan» de Hoogstraal, etc.

Elles sont, au contraire, décrites ou figurées par Sharif (1928), Toumanoff (1944), Hoogstraal et ses collaborateurs dans de nombreuses publications de 1959 à 1966, Arthur, « Tiques » (1960), Roberts (1968), etc.

Pomerantzev (1950) écrit à leur sujet: «Soies infra-internes... Celles-ci sont simples ou plumeuses, minces ou massives et peuvent servir comme caractères taxonomiques pour la détermination d'espèces ou de groupes d'espèces. »

Morel et Vassiliadès signalent (1962) un rapport entre la morphologie de ces soies et le cycle évolutif de certaines tiques.

Shatas (1956) place ces soies au premier rang des caractères distinctifs entre les larves et les nymphes de certaines espèces de Rhipicephalus. Le nombre et la structure des soies infra-internes lui permettent de séparer certaines espèces en deux groupes: Rhip. rossicus et Rhip. schulzei d'une part, $R h$. turanicus et $R$. sanguineus d'autre part. Le premier groupe possédant deux soies infra-internes à l'article I des palpes tandis que le deuxième groupe n'en possède qu'une au même article.

Pour toutes ces raisons, nous avons tenté de réunir les notions déjà acquises sur la morphologie de ces soies et les variations observées selon les espèces, le sexe et le stade d'évolution de divers Ixodidés. La présente note se bornera à rappeler les divers caractères généraux, surtout en ce qui concerne le genre. L'étude des espèces, à cet égard, sera tentée ultérieurement.

\section{Morphologie.}

a) Localisation. La figure 1 représente de façon demi-schématique les soies infra-internes d'un mâle de Dermacentor reticulatus articles I, II et III. En face les articles II et III d'un Haemaphysalis.

Le plus grand nombre des soies s'insère directement sur le palpe. Dans certaines espèces au contraire, l'insertion se fait soit sur une bandelette qui double le 
bord interne, soit, plus souvent, sur un appendice dépendant de l'article I, appelé «appendice sétigère»par certains auteurs et que nous appellerons la plaque. Cette plaque, comme appliquée sur la partie postérieure du palpe, est tantôt ovale, tantôt triangulaire.
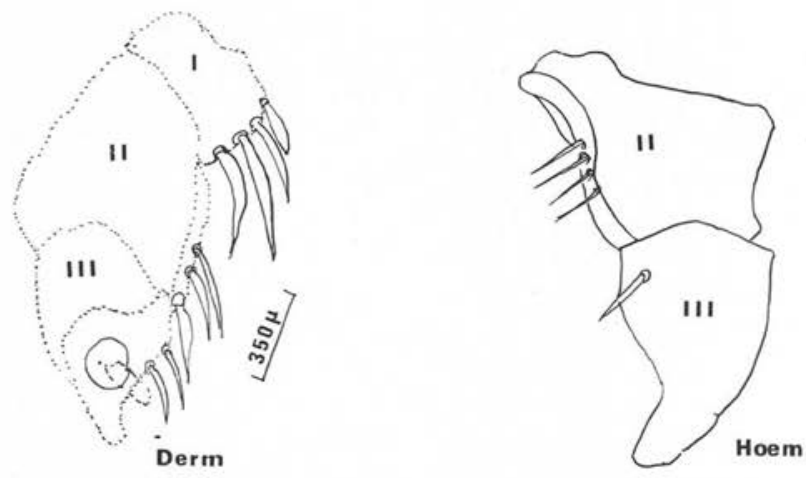

FIg. 1. - NI. Articles I, II et III de Dermacenoor reticulatus o et Haemaphysalis montrant l'insertion directe des soies sur le bord de l'article

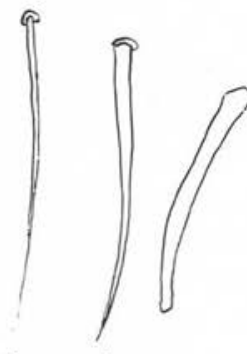

A $\quad$ B C
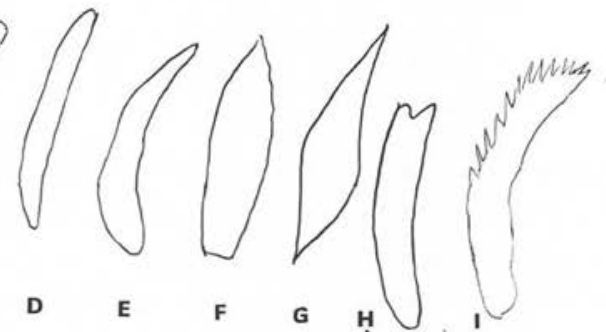

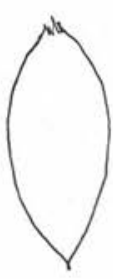

J

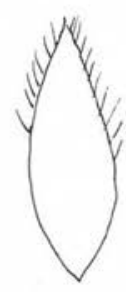

K

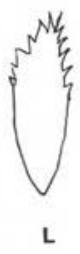

$\mathbf{L}$

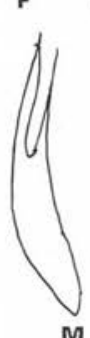

M
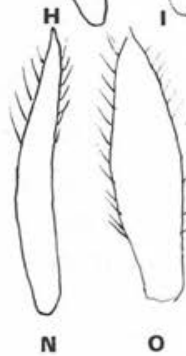

FIG. 2. - A, B, C, D, E, F, etc, montrant divers types de soies infra-internes. Leur appellation dans notre travail est indiquée dans le texte 
b) Forme DEs soies. Sur la même figure, au-dessous des précédentes, nous avons réuni divers types de soies:
(A) : soie simple,
(I) : courbe, denticulée,
(B) : simple mais plus épaisse et plus longue,
(J) : plus courte, en raquette,
(C) (D) : plus courte et plus épaisse. poin-
(K) : en ovale pointu,
tue ou non,
(E) : en croissant,
(F) : en fer de lance,
(G) : en «berlingot »,
$(\mathrm{H})$ : en doigt de gant,
(L) : en ovale plus court à apex pointu. denticulé ou non.
(M) : en ovale frangé,
(N): tubuliforme pointu,
(O) : fer de lance allongé et velu.

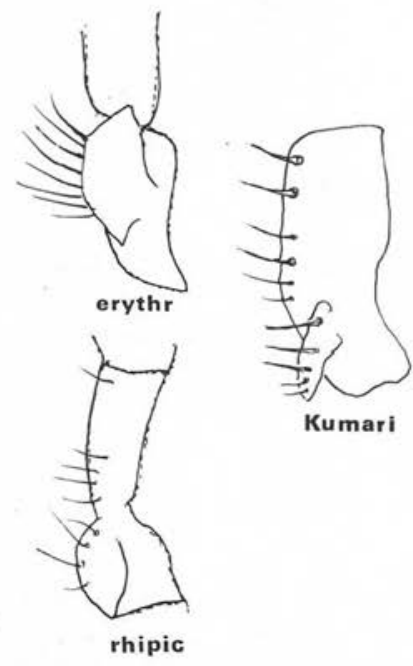

FIG. 3. - II. Soies infra-inter-

nes de $H$. erythraeam (erythr.) $H$.

Kumari (Kumari et $H$. rhipice-

phaloides (rhip.) montrant l'in-

sertion des soies de l'article I

sur une plaque

c) UNE FRANGE, plus ou moins fournie, s'observera donc chez certaines espèces. Elle sera parfois absente chez des espèces voisines.

d) LA LONGUeUR DE CES soies ne smble pas, pour l'instant, devoir fournir des caractères importants. On peut admettre, le plus souvent, une longueur moyenne de 50 à $70 \mu$.

e) Nombre. Le nombre des soies varie selon l'article considéré et, pour un même article selon le genre, le stade et le sexe.

Genres D'Ixodes. Dans le schéma précédent, nous avons figuré les soies d'un Dermacentor reticulatus mâle. L'article II (où les soies sont toujours présentes) porte trois soies. Dans d'autres genres, on trouverait par exemple:

Dermacentor: de 3 à 6 ,

Rhipicephalus: de 4 à 6 ,

Digeneus: de 2 à 4 ,

Haemaphysalis: de 2 à 7 ,
Amblyomma: de 2 à 4,

Hyalomma: de 4 à 9 ,

Ixodes: de 1 à 2 .

SEXE: Les sexes présentent parfois un dimorphisme très net. 
Article II

Mâles

Rhipicephalus : 4 (1 à 5)

Digeneus : 2 à 4

Dermacentor : 4 (4 à 6)

Haemaphysalis : 5 (3 à 10)

Ixodes: 2 (1 à 4$)$

Amblyomma : 5 (4 à 6)

Hyalomma : $3-4$ (3 à 9)

Anocentor: 4

Cosmiomma : $3-4$

Nosomma : 7 à 9
Femelles

6 (3 à 10)

4 à 5

5 (4 à 9)

6 (3 à 11)

3 (2 à 6)

3 (1 à 5)

6 (2 à 9)

4

3

8 (Sharif et ToumanofF)

Article I

Rhipicephalus : 5 (4 à 6)

7 (3 à 10)

Digeneus : 4 (3 à 5)

4 (4 à 5$)$

Dermacentor: 3 (3 à 5)

3 (3 à 6)

Haemaphysalis : 0 à 1 (0 à 2)

$0-1$ ( 0 à 2$)$

Ixodes: 0 ( 0 à 1$)$

0 (0 à 1) (2 ?)

Amblyomma : 2 (0 à 2)

2 (0 à 2)

Hyalomma : 4 (1 à 4)

4 (2 à 8)

Anocentor : 0

Cosmiomma : 1

0

1 (ARTHUR, 1920)

10 à 12 (SHARIF et TOUMANOFF)

Nosomma: 9 à 12

STADE: Outre les chiffres précédents valables pour les formes adultes nous trouvons pour les stades inférieurs (toutes espèces réunies).

LARVES :

Art. 1

Art. 2

Rhipicephalus:

5 fois 0

8 fois 1

5 fois 1

2 fois 2

LARVES :

Art. 1

Art. 2

Rhipicephalus :

5 fois 0

5 fois 1

Digeneus :

2 fois 0

Ixodes :

15 fois 0
8 fois 1

2 fois 2

1 fois 1

1 fois 2

9 fois 1

2 fois 2

3 fois 3 
Haemaphysalis :

3 fois 0

3 fois 1

Dermacentor :

1 fois 2

Amblyomma :

6 fois 0

6 fois 1

Hyalomma :

8 fois 0

1 fois 1

4 fois 2

Anocentor :

1 fois 0

1 fois 1

NyMPHES :

Art. 1

Art. 2

Rhipicephalus :

5 fois 1

5 fois 2

1 fois 2

1 fois 3

1 fois 3

Digeneus :

5 fois 1

2 fois 1

1 fois 3

Ixodes :

11 fois 0

8 fois 1

2 fois 1

5 fois 2

Haemaphysalis :

30 fois 0

9 fois 1

9 fois 2

5 fois 3

6 fois 4

Dermacentor :

1 fois 1

1 fois 1

Amblyomma :

11 fois 1

9 fois 1

1 fois 2

1 fois 3

Hyalomma :

6 fois 0

8 fois 1

6 fois 2

1 fois 3

Les chiffres précédents représentent le total de tous les chiffres publiés par divers auteurs (schémas) et de nos propres constatations. 
Sous forme plus condensée, les chiffres du tableau précédent (article I) pourront permettre une orientation (au moins schématique) vers les genres d'Ixodidés.

Mâles

0 ou 1 Haemaphysalis

0 ou 1 Ixodes, Margaropus

1 ou 2 Amblyomma

4 à 7 Hyalomma

7 Nosomma

8 ou 9 Rhipicephalus
Femelles

0 Haemaphysalis

0 ou 1 Ixodes, Amblyomma

1 (parfois 0) Margaropus-Boophilus

4 à 7 Hyalomma

5 à 10 Rhipicephalus

7 à 10 Nosomma

Nymphes

0 Haempahysalis

0 ou 1 Boophilus

1 ou 2 Rhipicephalus, Hyalomma-Ixodes-

Amblyomma

\section{Conclusions.}

Sans faire intervenir la diversité des formes, et en ne considérant que le nombre seul des soies infra-intetnes, nous voyons d'après les tableaux précédents:

$1^{\circ}$ que le nombre des soies va en croissant avec les stades successifs (ce qu'il était aisé de prévoir);

$2^{\circ}$ que ce nombre est légèrement plus élevé, au moins pour certains genres, chez les femelles que chez jes mâles;

$3^{\circ}$ que ces soies font absolument défaut sur l'article I dans quelques genres (Ixodes).

Cette dernière constatation permet de reconnaître à première vue les genres en question.

Une étude plus détaillée par genre et par espèce apportera peut-être un appoint à la diagnose habituelle.

\section{Bibliographie}

ARTHUR, 1963. - British ticks.

Feld-Musham, 1957. - Parasitology, 47 : 49-56.

FEIDER, 1965. - Arachnida, V (2).

Hoogstraial, 1959. - J. Parasitol., $45: 417$.

—, 1961. — Ibid., $47: 318$.

—, 1962. - Ibid., 48: 221.

—, 1964. — Ibid., 50: 789.

—, 1966. — Ibid., 52: 784, 789, 803.

-, 1963. - Ibid., 49.

— et KoHLs, 1959. - J. Parasit., $45: 417$.

— et —, 1968. — J. Parasit., $54: 1057$. 
— et -, 1965. - J. Parasit., 51: 460.

- et PARrish, 1968. - J. Parasit., $54: 616$.

- et -, 1968. - J. Parasit., $54: 402$.

-, Kohls et Trapido, 1965. — Ibidem, $51: 997$.

—, - et —, 1967. — Ibidem, 53: 197.

- , - et Parrish, 1967. - Ibidem, 53: 1096.

- et Trapido, 1963. - Ibidem, $49: 346$ et 489.

— et -, 1966. - Ibidem, 52: 1188.

-, - et KoHLs, 1965. - 51: 467 et 514 .

-, - et Rebello, 1963. - Ibidem, 49: 686.

-, Kniser and Coff Petersen, 1969. - Ann. Ent. Soc. Amer., 62: 415.

-, - - Ormsbee, Osborne, Helmy and Gaber, 1967. - J. Med. Ent., IV : 391.

- and MC CARTHY, 1965. - J. Parasit., 51: 674.

- and Wilson, 1966. - Ibid., 52: 614.

Kaster et Hoogstranl, J. Parasit., 1963, $49: 130$.

-, -, 1968. - Ann. Ent. Soc. America, 61 : 1228.

-, 一, 1964. - Acarologia, VI: 257.

Morel et Vassiliadès. - Rev. Elev., N. Série 1962: 343-386.

PomeranzeV, 1950. - Fauna of the U.R.S.S., p. 27.

Shatas, 1956. - Ent. Rev., 35 (4), 944. 